\title{
FACTORING FAMILIES OF POSITIVE KNOTS ON LORENZ-LIKE TEMPLATES
}

\author{
MICHAEL C. SULLIVAN
}

\begin{abstract}
We show that for $m$ and $n$ positive, composite closed orbits realized on the Lorenz-like template $L(m, n)$ have two prime factors, each a torus knot; and that composite closed orbits on $L(-1,-1)$ have either two for three prime factors, two of which are torus knots.
\end{abstract}

\section{INTRODUCTION}

The periodic orbits of a flow in a 3-manifold form knots. These knots and how they are linked have been studied with the aid of templates or knots holders, i.e. 2-dimensional branched manifolds with semi-flows [BW, GHS].

Let $m$ and $n$ be integers. The Lorenz-like templates, denoted $L(m, n)$, are shown in Figure 1. The semi-flow comes down from the branch line forming two bands. The left branch has $m$ half twists, while the right band has $n$. The sign convention for the twists is that left-handed twists are positive and right-handed ones are negative. The two bands meet tangentially at the branch line. $L(0,0)$ is called the Lorenz template. It arose as a model of the attractor studied by Lorenz [W1].

The semi-flow is uniformly expanding. The orbits which never exit the template constitute the invariant set of the semi-flow. The forward orbit of each point on the branch set that is in the invariant set is uniquely determined by an infinite sequence of ones and zeros. Speaking loosely, when an orbit wraps around the left band write a 0 , around the right band a 1 . A periodic orbit has a periodic symbol sequence. The inverse limit of the invariant set is the suspension of the full 2-shift. However, will shall not need symbolic dynamics except as a means to track orbits in this paper. See $[\mathbf{B W}]$ or $[\mathbf{G H S}]$.

As already noted a periodic symbol sequence determines periodic orbit. A periodic orbit however determines several sequences depending on the point on the orbit one starts with. Given a finite block or word of ones and zeros one can extend it to an infinite periodic sequence.

Date: June 20, 2007. 


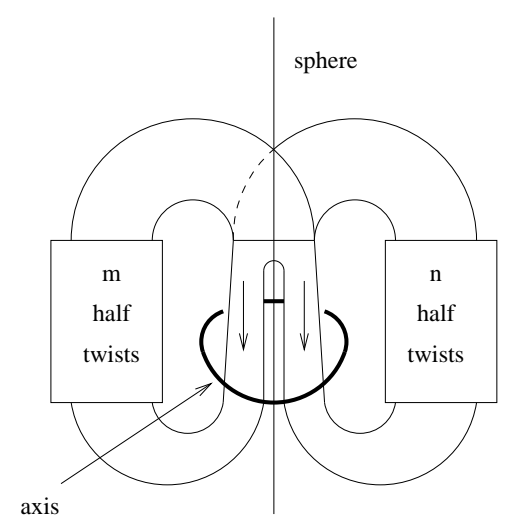

Figure 1. The Lorenz-like Template: the dark ring is the braid axis; the vertical line represents a factoring sphere.

Two nonperiodic blocks determine the same periodic orbit if and only if they are cyclic permutations. For example, $(00101)^{\infty}$ and $(01010)^{\infty}$ determine the same periodic orbit. (On $L(0,0)$ this orbit is seen to be a trefoil knot; see Figure 2.)

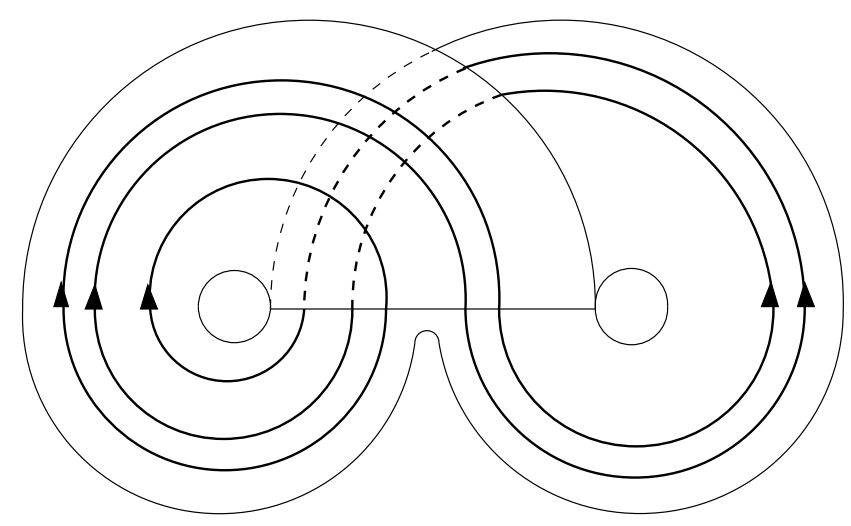

FiguRE 2. Lorenz template with a trefoil knot

The trip number of a periodic orbit on a Lorenz-like template is defined as follows. Among the nonperiodic blocks that generate a given periodic orbit choose the one that comes first in the dictionary ordering. Write it in the form $0^{m_{1}} 1^{n_{1}} \cdots 0^{m_{t}} 1^{n_{t}}$ where the powers are all positive. Then $t$ is the trip number. Thus, $(00101)^{\infty}$ has trip number two. Geometrically the trip number is the number of "trips" the orbit makes between the two bands. It is easy to see that all trip one Lorenz knots are trivial. 
The symbol $L(m, n)$ will also be used to represent the set of knots formed by the periodic orbits in the semi-flow of the template. Notice that $L(n, m)=L(m, n)$. It was shown in $[\mathbf{S} 1]$ that $L(0, n-2) \supseteq L(0, n)$, for all integers $n$, and that $L(0,-4) \supseteq L(0,-1)$.

In 1983 Bob Williams [W2] showed that for $n \geq 0$ all nontrivial knots in $L(0, n)$ are prime, but that $L(0,-1)$ contained a composite knot. It was shown in $[\mathbf{S 3}]$ that $L(0,-2)$ contains knots with arbitrarily many prime factors, and hence so too for $L(0, n)$ with $n<0$ by the set inclusions cited above. Ghrist $[\mathbf{G}]$ next showed that these templates contain all knots and links! In [S6] it was shown that if a template can be presented so that all the periodic orbits are positive braids, then there is bound of the number of prime factors of its periodic orbits. It is easy to see that $L(m, n)$ for $m, n>0$, can be so presented and that they have composite knots. Theorem 1 shows that the bound is two, far lower than given by the formula established in [S6].

The template $L(-1,-1)$ cannot be presented so that it's periodic orbits are positive braids, but it can be presented so that all its periodic orbits are positive knots [S5]. (The 5-knot is an example of a positive knot which is not a positive braid, although it is not known if the 5 -knot lives in $L(-1,-1)$.) In was conjectured in [S5] that knots in $L(-1,-1)$ can have at most two prime factors, however it turns out to be three (Theorem 2).

The major tools in our work are a theorem of Cromwell's $[\mathbf{C}]$ on the factoring of positive braids and its extension by Ozawa [O] to positive knots - Ozawa also greatly simplified Cromwell's proof. A knot diagram is irreducible if it has no cut points. Hence, a braid diagram of a nontrivial knot is reducible if and only if there is a generator that appears only once in the braid word. A knot diagram $D$ in a 2 -sphere is decomposable if there is a simple closed curve $C$ meeting $D$ just twice, transversely, such that neither $D \cap D_{1}$ or $D \cap D_{2}$ are arcs, where $D_{1}$ and $D_{2}$ are the two disks bounding $C$. If there is no such closed curve, the knot diagram is indecomposable. For a braid diagram on $s$ strands to be decomposable, its braid word must be conjugate to one of the form $a b$, where for some $1 \leq j<s-1, a$ is a word in the generators $\sigma_{1}, \ldots, \sigma_{j}$, and $b$ is a word in $\sigma_{j+1}, \ldots, \sigma_{s-1}$. Any such value $j$ - there could be several - will be called a factoring site.

Theorem (Cromwell-Ozawa). Let $D$ be a positive irreducible knot diagram for $K$. Then $K$ is prime if and only if $D$ is indecomposable. 


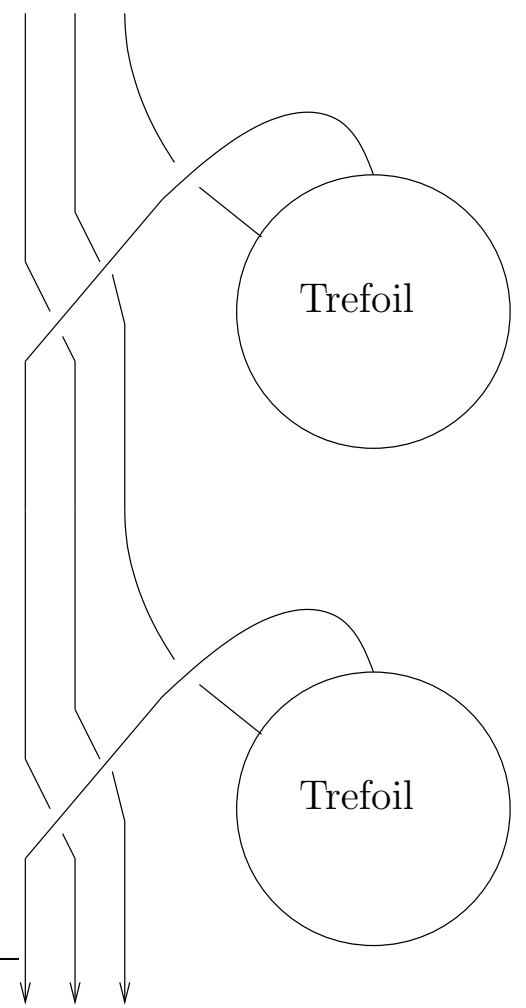

FiguRE 3. Use the half twist to create two more trefoils for a total of three prime factors.

$$
\text { 2. } L(m, n)
$$

Nontrivial torus knots are known to be prime.

Theorem 1. For $m$ and $n$ positive, composite knots on $L(m, n)$ have two prime factors each of which is a torus knot.

Proof. Trip one knots are factored by the sphere indicated in Figure 1. The left factor can be placed as a trip one knot in $L(m, 0)$ and the right factor can be placed as a trip one knot in $L(0, n)$. It was shown in [S6, Lemma 3.1] that trip one knots in $L(0,1)$ are torus knots. The proof is easily modified for $L(0, n)$ with $n$ positive and odd. For $n$ even the proof is immediate: the orbit $\left(01^{p}\right)^{\infty}$ has braid word $\sigma_{p-1} \cdots \sigma_{1} \Delta^{n}$ and can be placed on torus as $T_{p, 1+p n / 2}$. ( $\Delta$ represents a half twist of all strands.) This factoring could result in one or both factors being trivial. Thus, trip one knots on $L(m, n)$ are either trivial, torus knots, or the connected sum of just two torus knots.

Let $K$ be a knot in $L(m, n)$ with trip number $t \geq 2$; let $K$ 's braid diagram have $p$ strands going down the left band and $q$ strands going 
down the right band. If $m, n>1$, then there are more than one of each of the generators $\sigma_{1}, \ldots, \sigma_{p-1}$ in the twists of the left band, and more than one of each of the generators $\sigma_{p+1}, \ldots, \sigma_{p+q-1}$ in the twists of the right band. And, there are $t \geq 2 \sigma_{p}$ 's in $K$ 's braid word. Thus, the diagram is irreducible.

Suppose the braid diagram of $K$ is decomposable with $j$ a factoring site. Let

$$
g=\left(\sigma_{p} \sigma_{p+1} \cdots \sigma_{p+t-1}\right)\left(\sigma_{p-1} \cdots \sigma_{p+t-2}\right) \cdots\left(\sigma_{p-t} \cdots \sigma_{p}\right) .
$$

The braid word for $K$ can be written as $g a \Delta_{R}^{m} b \Delta_{L}^{n}$ where $a$ is a word in the first $p-1$ generators, $\Delta_{R}$ is a half twist of the first $p-1$ generators, $b$ is a word in the last $q-1$ generators, and $\Delta_{L}$ is a half twist on the last $q-1$ generators. See Figure 4 .

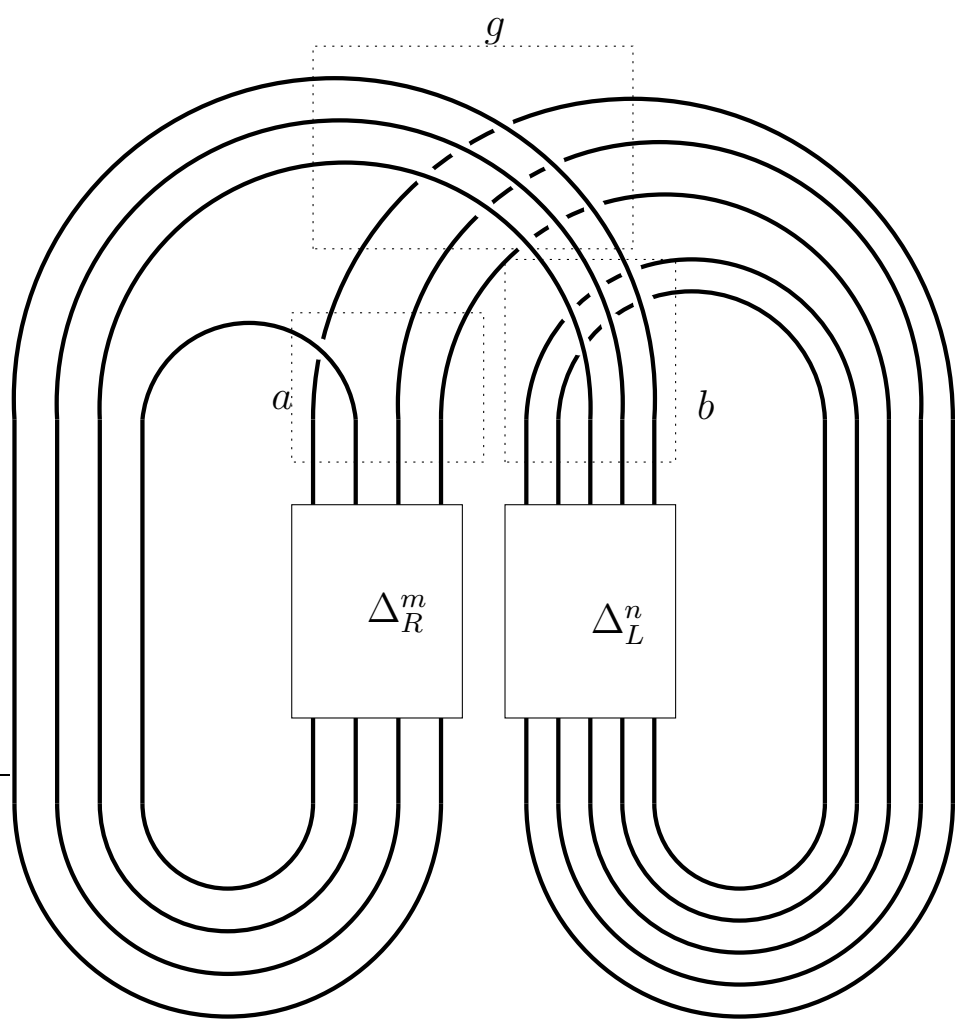

Figure 4. Braid word

There are $t$ occurrences of $\sigma_{p} \sigma_{p+1}$ in $g$. Thus, $j \neq p$.

Suppose, $j \leq p$. The completion of the braid word $a \Delta_{R}^{m}$ may produce a link. But, we can add (concatenate) a word $g^{\prime}$ to the beginning that permutes the last $t$ strands in the same manner that the corresponding 
strands of the original knot were permuted by their transit around the left band. Thus, $g^{\prime} a \Delta_{R}^{m}$ gives a knot. By $[\mathbf{S} 4]$ positive braids with a half twist are prime (this can also be deduced directly from Cromwell's Theorem). But, this is a contradiction.

Similarly, one can show $j$ is not greater than $p$. Hence, for $m, n>1$ the proof is complete. When $m$ or $n$ is one, we have not shown the braid is irreducible.

Suppose $m=1$. Then there may be only one occurrence of $\sigma_{1}$ in the braid word. If this is the case, one can apply an $R_{1}$ Reidemeister move and eliminate this crossing. Even if $n=1$ it is still the case that there is more than one occurrence of $\sigma_{p+1}$ in the braid word, since $\sigma_{p+1}$ occurs in $g$. Thus we have an irreducible braid. The analysis above can be redone with slight modifications to complete the proof.

$$
\text { 3. } L(-1,-1)
$$

Observation. If a braid diagram is irreducible and indecomposable, then it remains so if additional crossings are added, fixing the number of strands. Thus, if a prime knot is presented by a positive irreducible indecomposable braid diagram and some extra positive crossings are added so that the new diagram still represents a knot, this new knot is prime.

Theorem 2. Composite knots on $L(-1,-1)$ have either two or three prime factors. If there are three prime factors, they are all torus knots. If the are two prime factors at least one is a torus knot.

Proof. In $[\mathbf{S 5}]$ the template $L(-1,-1)$ was shown to be equivalent to the template in Figure 5, which we shall dub $H H$. By equivalent we mean that a link is in $H H$ if and only if it is in $L(-1,-1)$. The equivalence is shown a performing a type of surgery that does not effect invariant orbits followed by an ambient isotopy. The knots in $H H$ are presented as having only positive crossings, but many are not braided. We work with $H H$ from now on. The reader should notice that $H H$ is a double branched covering of $L(0,1)$ - often called the horseshoe template as it tracks the orbits of a suspension of Smale's famous horseshoe map.

We establish some notation. Let $K$ be a given knot in $H H$. The two dashed rectangles in Figure 5 represent spheres. (We will eventually show that any composite knot on $H H$ is factored by one or both of these spheres.) Let $t_{1}$ denote the number of times $K$ enters the top sphere, and let $t_{2}$ denote the number of times $K$ enters the bottom sphere. (We will eventually show that if $t_{1}$ and $t_{2}$ are greater than one, then $K$ is prime.) 
Suppose $t_{1}=t_{2}=1$. It is easy to see that the two spheres of Figure 5 factor $K$. Two of the factors, the ones inside the spheres, are trip one $L(0,1)$ knots and hence are torus knots, which could be trivial. The external factor, if it is not trivial, has braid word $\left(\sigma_{p-1} \cdots \sigma_{p-i}\right)\left(\sigma_{p-1} \cdots \sigma_{p-j}\right)$, for some $p, i$ and $j$. But, either $i$ or $j$ must be $p-1$; without loss of generality assume $i=p-1$. We can apply $R_{1}$ moves until we have a braid word of the form $\left(\sigma_{j} \cdots \sigma_{1}\right)^{2}$; here we have reindexed the braid group generators. This diagram is irreducible and indecomposable, hence the external factor is prime. It is also easy to see that it is the torus $\operatorname{knot} T_{j, 2}$.

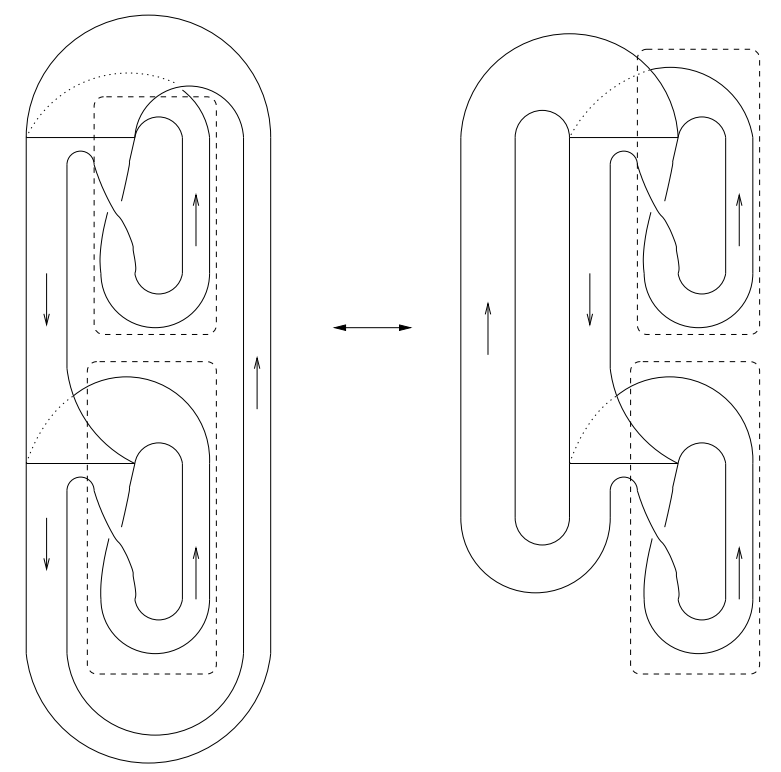

Figure 5. The template $H H$

Now suppose $t_{1}=1$ but $t_{2}>1$. The upper sphere still factors. The external factor can be braided. It's braid word is a concatenation of an $L(0,1)$ knot with $\sigma_{p-1} \cdots \sigma_{p-j}$. By the Observation it is prime if nontrivial. The case $t_{2}=1$ and $t_{1}>1$ is similar.

Before we consider the case $t_{1}, t_{2}>1$ we make some general remarks. Given an irreducible positive diagram how do we check that it is indecomposable? Think of the diagram as a graph with the double points as vertices and the arcs connecting them as oriented edges. If there is a curve $C$ that factors the diagram (in the sense of Cromwell-Ozawa Theorem) then it meets the diagram transversely in two non-double points. Thus there are two regions in the compliment of the diagram that that border along two distinct edges. One can just check each 
region to see if any adjoining regions satisfy this requirement. If not, the knot in question is prime. In the standard rendering of the trefoil knot there are just five regions to check. None shares more than one edge with another. Thus the trefoil is prime. Figure 6 shows a knot on $H H$, or rather its diagram; we have not shown the crossing types since they are all positive. Applying one $R_{1}$ move makes it irreducible. The reader can then check each of the 23 regions and show the knot is prime.

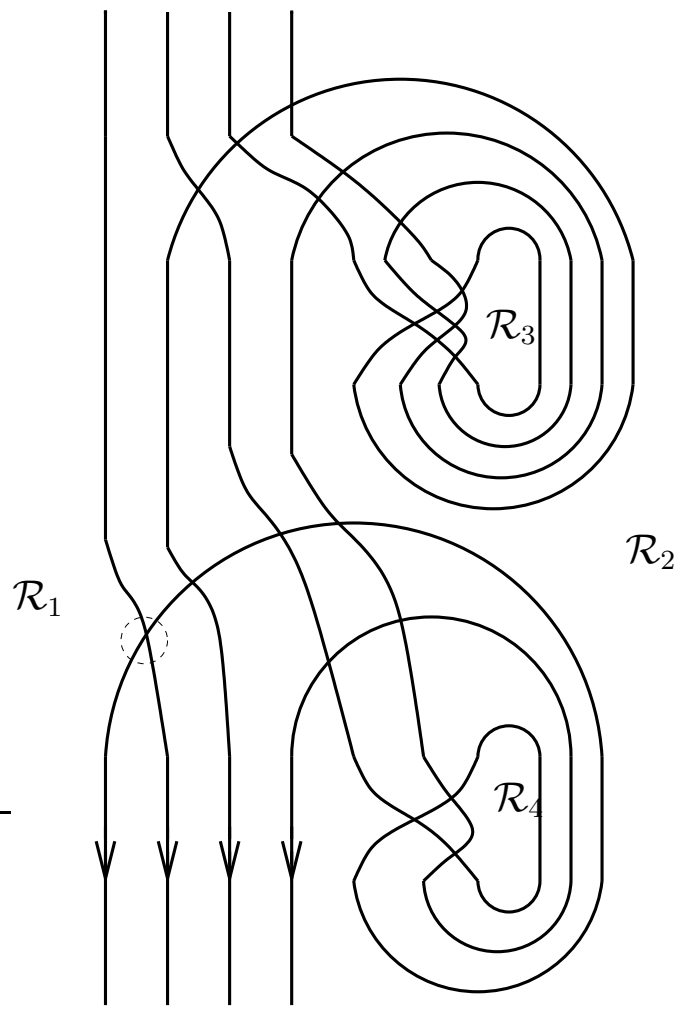

Figure 6. A knot in $H H$

Let $K \in H H$ with $t_{1}, t_{2}>1$. Let $D(K)$ be the diagram given by projecting $K$ onto the plane with $H H$ as in the left hand version of $H H$ shown in Figure 5.

As we have seen it may be the case that $K$ is reducible. We claim: any cut point in $D(K)$ occurs when one of the outer most arcs returning to the annulus from a half twist crosses over a strand on the annulus; only one such arc creates cut points; and they can be removed by a sequence of $R_{1}$ moves. We assume this for now and prove it later as 
Lemma 5. This proof, although not hard, uses terminology developed below and is logically independent.

Lemma 3. Regions with 2, 3, or 4 edges cannot be factoring regions.

Proof. Suppose $C$ meets a 2-gon. It must cross each edge. (Otherwise there would be a trivial factor.) But then both the vertices would have to have been points for the original diagram.

Suppose $C$ meets a triangular region. It must cross two of the edges. But then the common vertex of these two edges would have to have been a cut point for the original diagram.

We claim any rectangular region in $D(K)$ will have opposite edges with parallel orientations. A half-twist on $n$ strands has $\sum_{i=1}^{n-3} i$ 4sided regions each in the described form. Any other 4-sided regions are formed from two arcs of $K$ from the non-twisted bands on top, with parallel orientations, and two arcs of $K$ coming around from a half-twist, with parallel orientations. Since all four crossings need to be positive, rectangular regions can only have the specified form. If $C$ meets such a region it must meet opposite edges by irreducibility otherwise we would get a cut point. But then $K$ pierces the would-be factoring sphere twice from the same side, a contradiction.

We now define four regions, $\mathcal{R}_{1}, \ldots, \mathcal{R}_{4}$ in $D(K)$ for special consideration. Let $\mathcal{R}_{1}$ be the unbounded region (using the left hand version of $\mathrm{HH}$ in Figure 5). Let $\mathcal{R}_{3}$ be the region that contains the upper relative braid axis and let $\mathcal{R}_{4}$ be the region that contains the lower relative braid axis. Let $\mathcal{R}_{2}$ be the region that contains the center of the outer boundary circle of $H H$. Figure 6 gives an example.

Lemma 4. The regions $\mathcal{R}_{1}, \ldots, \mathcal{R}_{4}$ are not factoring regions.

Proof. Whether or not $R_{1}$ moves are needed to make $D(K)$ irreducible, $\mathcal{R}_{1}$ has two vertices in its boundary. So, it only borders two regions, each along a single edge. Thus, it does not border any region twice.

One can check that $\mathcal{R}_{2}, \mathcal{R}_{3}$ and $\mathcal{R}_{4}$ do not border any region twice.

To conclude the proof of Theorem 2 we divide the diagram up into zones.

Type-0: We define a zone that joins the bottom of a half twist and then loops around. There will be two possible subtypes depending on the diagram for $K$.

Subtype-0a: If the right most strand coming out of the half twist meets the right most of the $t_{i}$ strands coming into the half twist from the annulus, a Subtype-0a zone is formed. If $q_{i}$ is the number of strands 
in the half twist there will be $q_{i}-24$-sided regions and one 3-sided region. The knot in Figure 6 has two such zones.

Subtype-0b: See Figure 7. The other possibility is that the right most strand coming out of the half twist returns first to the half twist. Now we have $q_{i}-34$-sided regions, one 5 -sided region and one 2 -sided region. The 5-sided shares two edges with regions in the half twist; we will see below that no region in the half twist has more than four sides. The 5-sided region shares two edge with 4 -sided regions within its zone. Thus, the 5-sided region cannot border another five or higher sided region twice and hence cannot be a factoring region.

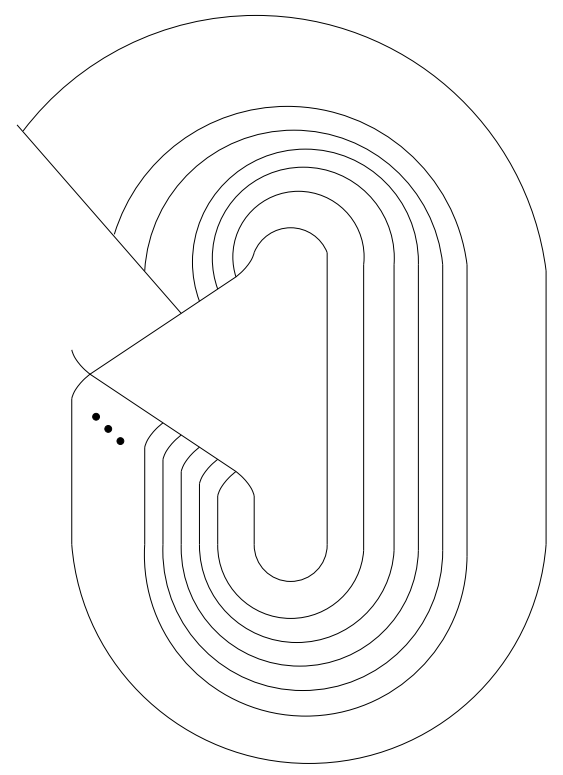

Figure 7. A zone of subtype-0b

Type-I: Two type-I zones are formed when each of the $t_{i}(i=1,2)$ strands leaving the annulus and the respective $t_{i}$ strands returning cross. These zones have only 4 -sided regions.

Type-II: Type-II zones are the roughly triangular zones formed by the half twists. More precisely, they are bounded by the two arcs that cross all the way across the half twists and the disks $\mathcal{R}_{3}$ or $\mathcal{R}_{4}$. Let $p$ be the number of strands going through a half twist. Then the zone of type-II has $p-23$-sided regions and $\Sigma_{i=1}^{p-2} i$-sided regions and no others.

Type-III $\&$ and its variants: We pause to make some general geometric observations. Let $A B C D$ be a rectangle with $A$ as its upper left corner, $B$ as its upper right corner, etc. Select a point $E$ in the interior 
of the $B C$. Place a fifth line segment starting from $E$ and going down as we go from right to left. Assume this segment misses $D$. If it meets the interior of $D A$ the rectangle we be partitions into two 4-sided regions. If the new line segment meets the interior of $C D$ the rectangle is partitioned into a 5-gon and a triangle. See Figure 8. Now suppose we have selected $n$ points in the interior of $B C: E_{1}, \ldots, E_{n}$. Draw a line segment from each going down as we go left. Assume no segment meets $D$ and all are disjoint. Then there is at most one 5-sided region and it must border $C D$. The proof is by induction on $n$.
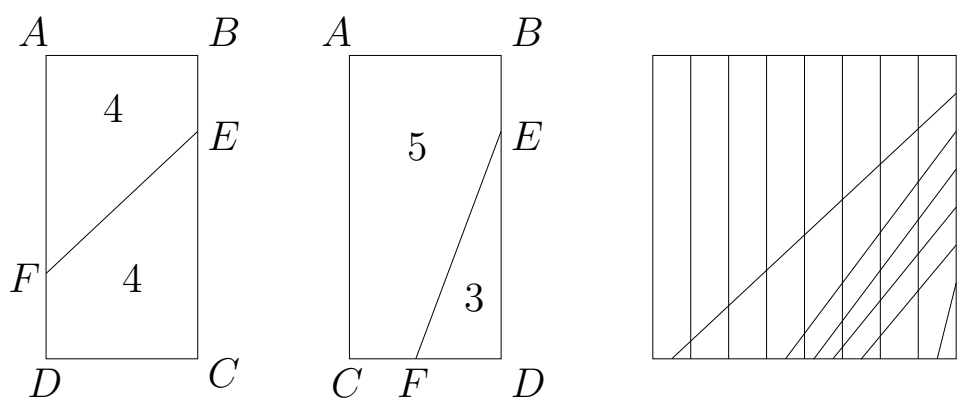

Figure 8. At most five sided regions can be formed.

Now suppose the rectangle $A B C D$ is also partitioned by $m$ vertical segments from $A B$ to $C D$. By induction on $m$ one can show that each of the $m-1$ vertical strips has at most one 5-sided region, that these do not border each other along an edge and that only the left most 5 -gon borders the segment $A B$. All 5 -gons border $C D$. This structure will be call a zone of type-III. If a corner vertex is rounded off, we get a zone of type-III'. The same observations apply to zones of type-III'.

We return to the analysis of the knot $K$.

Just above each half twist and below the zones of type-II are some additional regions. See Figure 9. They can be deformed to rectangular figures with $t_{i}-1$ vertical strips with some line segments crossing downward from the right. We get a zone of type-III or type-III' depending of whether the inner most strand coming out of the half twist crosses any of the $t_{i}$ strands coming from the annulus or it goes directly back into the half twist, respectively. Above, below and to the right we have shown there are no regions with more than 4 sides, and we have already shown $\mathcal{R}_{2}$ is not a factoring region. Thus, there are no factoring regions in these two zones.

There are two more zones to consider. They are on the annulus. Their precise definition depends on certain features of the knot $K$. 


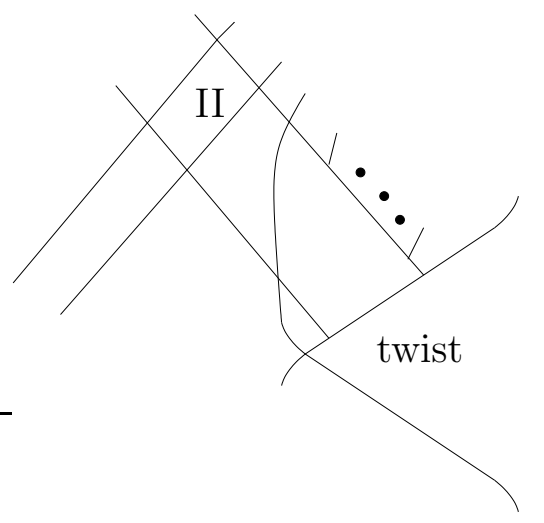

FiguRE 9. Intermediate zone

We divide this description into three cases. (Case B below has two subcases.)

Case A: Both the outer most arcs coming from the Möbius bands to the annulus cross all strands. Then the zones between these arcs are as depicted in Figure 10. The rectangle $A B C D$ is a type-III zone. It is easy to show that the 5-gon $B F G H C$ is partitioned into some number of 4-gons and a single 5-gon that cannot border another 5-gon along an edge. The triangle $A D E$ has no 5-gons.

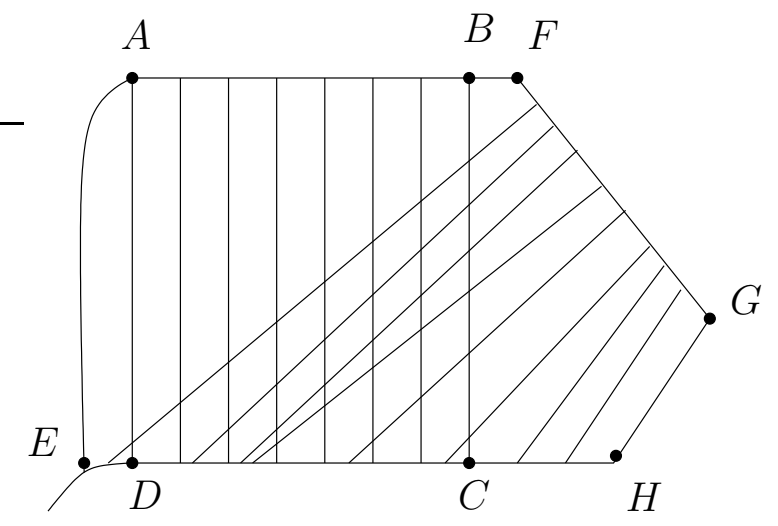

Figure 10. A zone with a type-III' zone inside

Case B: One of the outer most strands coming from the Möbius bands must cross all the strands on the annulus or else the diagram will not be connected. In the case that only one does we have an additional type of zone to consider; we dub it the annular zone. The analysis is essentially the same as for the type-III zones, but some extra 
fiddling is required. We shall first study the knot $K$ as it appears on the template $H H$. At the end we show that the effect on our analysis of any $R_{1}$ moves needed to make the diagram irreducible is benign.

Without loss of generality assume the outer most strand of the lower Möbius band crosses all the strands on the annulus and that the outer most strand on the upper Möbius band does not. This strand will come down and cross the lower branch line (really we work with the projections of the branch lines). Either it will wind around on the annulus or go immediately to the lower Möbius band. This gives two subcases for the form of the annular region, but the analyzes are nearly identical.

Figure 11 shows an annular zone. The outer circle is former from the arcs from $B$ to $C$ and from $C$ to $B$. Let $n$ strands cross over from the lower Möbius band and then $n+1$ exit the just above the upper branch line. In Figure 12 we cut open the annulus along the arc $A B$ creating a rectangle $A B C A^{\prime}$ with a distinguished point $B^{\prime}$ between $C$ and $A^{\prime}$. The resulting figure allows us to see that the conclusions arrived at for the type-III zones hold, except that there may be a single 6-gon, because of $B^{\prime}$, and we may have two strands coming out of $A$. Let $q$ be 5 or 6 . It is still the case that none of the $q$-gons border each other on an edge. If a $q$-gon in an annular zone should border and q-gon in another zone, it does so along at most one edge: but a factoring region must border another factoring region along at least two edges. The effect of applying $R_{1}$ moves is only to reduce the number of regions.

This concludes the proof of Theorem 2 .

Lemma 5. Any cut point in $D(K)$ occurs when one of the outer most arcs returning to the annulus from a half twist crosses over a strand on the annulus; only one such arc creates cut points; and they can be removed by a sequence of $R_{1}$ moves.

Proof. A cut point can only occur at a point where a region touches itself. Because $t_{1}, t_{2}>1$ we see that $\mathcal{R}_{2}$ is not such a region. It is clear that regions $\mathcal{R}_{1}, \mathcal{R}_{3}$ and $\mathcal{R}_{4}$ are not such regions. There are not any self-connecting regions in zones of type-0, 1 , or 2 . We now consider crossing that occur over strands that stay in the annulus. Index the gaps between strands as is done with braids. If both outer most arcs returning to the annulus from the half twist cross over index $i$ no selfconnecting regions will be formed. If one of these arcs goes out farther than the over, we will get cuts, but only of the type described in the lemma. 


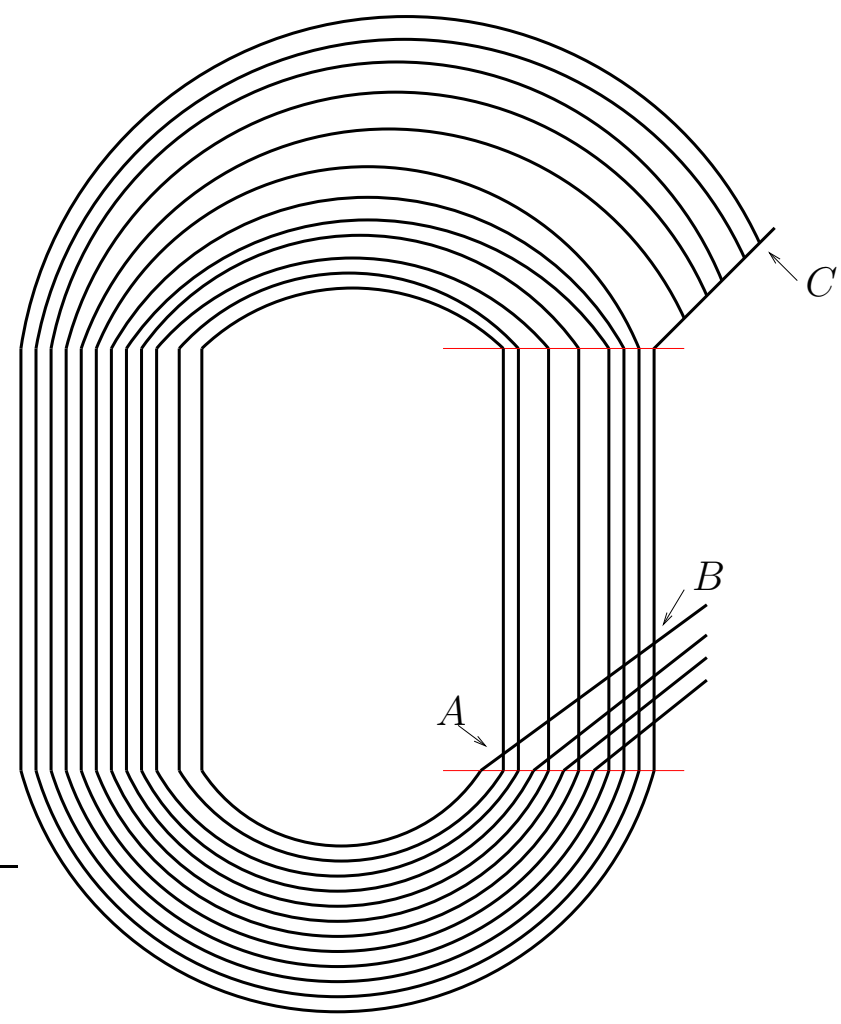

Figure 11. An annular zone

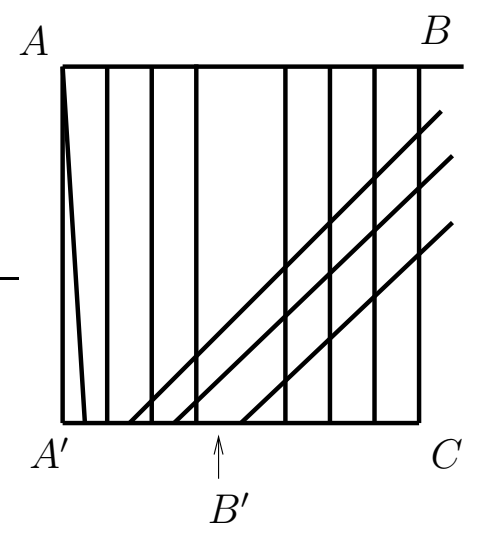

FiguRE 12. An annular zone cut open 


\section{REFERENCES}

[BW] J. Birman \& R. F. Williams. Knotted Periodic Orbits in Dynamical Systems I: Lorenz Knots. Topology 22 (1983), 47-82.

[C] P. Cromwell. Positive braids are visually prime. Proc. London Math. Soc. (3) 67 (1993) 384-424.

[G] R. Ghrist. Branched two-manifolds supporting all links. Topology 36 (1997), no. 2, 423-448.

[GHS] R. Ghrist, P. Holmes \& M. Sullivan. Knots and links in Three-Dimensional Flows, Lecture Notes in Mathematics, Vol. 1654, Springer-Verlag, Berlin, 1997.

[O] M. Ozawa. Closed incompressible surfaces in the complements of positive knots. Comment. Math. Helv. 77 (2002), no. 2, 235-243.

[R] C. Robinson. Homoclinic bifurcation to a transitive attractor of Lorenz type. Nonlinearity, 2:495-518, 1989.

[S1] M. Sullivan. Prime decomposition of knots in Lorenz-like templates, J. Knot Theory Ramifications 2 (1993) 453-462.

[S3] M. Sullivan. The prime decomposition of knotted periodic orbits in dynamical systems, J. Knot Theory Ramifications 3 (1994) 83-120.

[S4] M. Sullivan. Positive braids with a half twist are prime, Journal of Knot Theory and its Ramifications, Vol. 6, No. 3, (1997) 405-415.

[S5] M. Sullivan. Positive knots and Robinson's attractor, The Journal of Knot Theory and its Ramifications, Vol. 7, No. 1, (1998) 115-121.

[S6] M. Sullivan. Knots on a Positive Template have a Bounded Number of Prime Factors. Algebraic and Geometry Topology, 5 (2005), paper no. 24, 563-576.

[W1] R. F. Williams. The Structure of Lorenz Attractors. Turbulence Seminar, Lecture Notes in Mathematics, Vol. 615, ed. by A Dold \& B. Eckmann, Springer-Verlag, (1976) 94-112.

[W2] R. F. Williams. Lorenz knots are prime. Ergod. Th. \&3 Dynam. Sys. 4 (1983) $147-163$.

Department of Mathematics (4408), Southern Illinois University, Carbondale, IL 62901, USA, msulliva@math.siu.edu, http://www.math.siu.edu/sullivan 